\title{
Individual differences in theta-band oscillations in a spatial memory network revealed by electroencephalography predict rapid place learning
}

Brain and Neuroscience Advances Volume 5: 1-17

(C) The Author(s) 2021

Article reuse guidelines:

sagepub.com/journals-permissions DOI: $10.1177 / 23982128211002725$ journals.sagepub.com/home/bna

(S)SAGE

\author{
Markus Bauer $^{1}$ (i), Matthew G. Buckley² ${ }^{\text {iD }}$ and Tobias Bast ${ }^{1}$
}

\begin{abstract}
Spatial memory has been closely related to the medial temporal lobe and theta oscillations are thought to play a key role. However, it remains difficult to investigate medial temporal lobe activation related to spatial memory with non-invasive electrophysiological methods in humans. Here, we combined the virtual delayed-matching-to-place task, reverse-translated from the watermaze delayed-matching-to-place task in rats, with highdensity electroencephalography recordings. Healthy young volunteers performed this computerised task in a virtual circular arena, which contained a hidden target whose location moved to a new place every four trials, allowing the assessment of rapid memory formation. Using behavioural measures as predictor variables for source reconstructed frequency-specific electroencephalography power, we found that inter-individual differences in 'search preference' during 'probe trials', a measure of one-trial place learning known from rodent studies to be particularly hippocampus-dependent, correlated predominantly with distinct theta-band oscillations (approximately $7 \mathrm{~Hz}$ ), particularly in the right temporal lobe, the right striatum and inferior occipital cortex or cerebellum. This pattern was found during both encoding and retrieval/expression, but not in control analyses and could not be explained by motor confounds. Alpha-activity in sensorimotor and parietal cortex contralateral to the hand used for navigation also correlated (inversely) with search preference. This latter finding likely reflects movement-related factors associated with task performance, as well as a frequency difference in (ongoing) alpha-rhythm for high-performers versus low-performers that may contribute to these results indirectly. Relating interindividual differences in ongoing brain activity to behaviour in a continuous rapid place-learning task that is suitable for a variety of populations, we could demonstrate that memory-related theta-band activity in temporal lobe can be measured with electroencephalography recordings. This approach holds great potential for further studies investigating the interactions within this network during encoding and retrieval, as well as neuromodulatory impacts and age-related changes.
\end{abstract}

\section{Keywords}

Memory, electroencephalography, theta oscillations, water maze, medial temporal lobe

Received: 5 January 2021; accepted: 24 February 2021

\section{Introduction}

A large body of research in humans and animal models supports the view that the hippocampus and the surrounding medial temporal lobe (MTL) cortices play a crucial role in certain types of memory, including place memory (Bast, 2007; Burgess et al., 2002; Morris, 2007). Theta oscillations are a prominent neural activity pattern recorded from MTL regions, in particular the hippocampus, especially during movements in real and virtual environments. Theta oscillations have been associated with encoding and retrieval of memory, including place memory, and this has been suggested to reflect that theta oscillations facilitate underlying synaptic plasticity mechanisms, separate encoding and retrieval processes, or coordinate the activity of neuronal ensembles that are distributed across hippocampus and other brain regions and interact to support memory encoding, retrieval or expression (Colgin, 2013; Cornwell et al., 2008; Hasselmo and
Stern, 2014; Herweg et al., 2020; Kahana et al., 1999; O'Keefe and Burgess, 1999). The evidence linking theta oscillations with these memory processes has traditionally come from invasive recordings in animals and non-invasive recordings in humans, but recent studies have further supported this evidence with

\footnotetext{
${ }^{1}$ School of Psychology and Neuroscience@Nottingham, University of Nottingham, Nottingham, UK

${ }^{2}$ Department of Neuroscience, Psychology and Behaviour, University of Leicester, Leicester, UK

\section{Corresponding authors:}

Markus Bauer and Tobias Bast, School of Psychology and

Neuroscience@Nottingham, University of Nottingham, University Park, Nottingham NG7 2RD, UK.

Emails: markus.bauer@nottingham.ac.uk; tobias.bast@nottingham.ac.uk
} 
intracranial recordings obtained directly from the human hippocampal formation, for example, Bush et al. (2017).

Watermaze tests of place learning and memory in rodents, and corresponding reverse-translated human paradigms in real or virtual environments, have long been used to study hippocampal function (Buckley and Bast, 2018; Cornwell et al., 2008; Morris, 2007; Morris et al., 1982; Pu et al., 2017). In common variants, the rodent or human participant has to find a hidden goal that remains in the same place over many trials, allowing for incremental learning of the place with reference to distal cues surrounding a circular, featureless maze. Place memory of where the goal is located in relation to the distal cues (i.e. allocentric place memory) is reflected by relatively short latencies and direct paths to the goal when the animals are placed into the maze from different start positions (which discourages use of egocentric strategies), and by search preference, that is, persistent searching around the goal location, when the goal has been removed for a probe trial. Although incremental place-learning performance depends on the hippocampus, rodent studies have shown that one-trial place-learning performance, as measured using the delayed-matching-to-place (DMP) watermaze variant, is a more sensitive index of hippocampal function (reviewed in Buckley and Bast (2018)).

The DMP task requires the continuous rapid updating of place memory, resembling the everyday task of, for instance, remembering where we parked our car on a particular occasion. In a common DMP protocol (Bast et al., 2009; Steele and Morris, 1999), which we have recently reverse-translated into a virtual DMP task for human participants (Buckley and Bast, 2018), the goal moves to a new place every four trials. One-trial place learning on the rodent and human DMP task is reflected by marked reductions in latency and path lengths to the goal from trial 1 to 2 , with little further improvements on subsequent trials, and by a marked search preference for the vicinity of the goal location when trial 2 is run as probe (when the goal is removed). Rodent studies have shown that such one-trial place-learning performance is highly hippocampus-dependent, being more sensitive to disruption of hippocampal function than incremental place-learning performance; for example, partial hippocampal lesions and manipulations of hippocampal synaptic plasticity, which can leave incremental place-learning performance relatively intact, markedly impair one-trial place-learning performance (reviewed in Buckley and Bast (2018)). Moreover, the DMP task allows for the repeated study of encoding and retrieval/expression of new place memory within the same participants. Thus, the virtual DMP task may be particularly suitable to reveal the neural oscillations associated with encoding or retrieval/expression of hippocampus-dependent place memory performance in human participants.

There is considerable inter-individual variance in cognitive performance parameters. For instance, ageing affects spatial memory in particular and this may happen through decline of cholinergic neuromodulation (Richter et al., 2014). In Alzheimer's disease (AD), the MTL and the nucleus basalis are among the first brain areas to be damaged (Braak and Braak, 1995), and several studies have shown that a deficit in spatial memory in AD correlates with hippocampal damage (Kramer et al., 2004; Miller, 1973). We have previously shown that functional connectivity of the nucleus basalis to cortex can predict the therapeutic response to cholinesterase inhibitors by patients suffering from mild cognitive impairment; more specifically, functional connectivity of the nucleus basalis predicted the improvement of cognitive performance scores in response to treatment with cholinesterase inhibitors (Meng et al., 2018). A question that arises is, how do such cholinergic changes mediate memory processes? One candidate mechanism is through hippocampal theta oscillations as these are known to be dependent on cholinergic input (Newman et al., 2012; Tiesinga et al., 2001). The virtual DMP task might therefore serve as an ideal model to study such mechanisms in the future.

In this study, we aimed to examine whether electroencephalography (EEG) was suitable to measure memory-related theta oscillations from the MTL in human participants performing the virtual DMP test. Previous studies have used magnetoencephalography (MEG) to localise spatial navigation-related theta oscillations (Cornwell et al., 2008; Kaplan et al., 2014; Kaplan et al., 2012; Pu et al., 2017), but MEG is far more expensive than EEG and these authors also used individual anatomical magnetic resonance imagings (MRIs) of the head to construct appropriate volume conductor (and thus biophysical forward) models for source analysis, making this method much less commonly available to use for future studies of individual differences in the context of ageing or other clinically relevant conditions or in particular diagnostic tests for early dementia. MEG is also particularly more sensitive to more superficial sources (Hillebrand and Barnes, 2002) and might therefore be, relatively spoken, less advantageous to study deeper sources. In addition, we aimed to perform a largely data-driven analysis to reveal key frequency bands (within the range of $2-15 \mathrm{~Hz}$ ) and brain regions most robustly associated with performance, rather than constraining our analysis to predetermined frequencies or regions of interest.

\section{Materials and methods}

\section{Participants}

Initially, 26 participants were recruited, predominantly students from the University of Nottingham recruited through an opportunity sample as part of MSc summer research projects. The study was run in line with ethical guidelines and approval for taught research projects was obtained from the Ethics Committee of the School of Psychology, University of Nottingham. All participants gave informed consent. Participants were given an inconvenience allowance for their participation. The sample size was chosen to represent a compromise between statistical power and practical considerations. It was a little higher than sample sizes in previous MEG studies of spatial memory and navigation-related theta (18 participants ( $\mathrm{Pu}$ et al., 2017); 19 participants (Kaplan et al., 2012)). The data of five participants had to be excluded due to problems with their EEG data, either trigger problems or electrical noise issues caused by an electrical device on the roof of the semishielded room in which the experiment was conducted. The data of 21 participants ( 15 female and 6 male) were thus included in this report (mean age $=24.4$ years, standard deviation $=2.9$ years ).

\section{Virtual DMP task}

The virtual DMP task was run using MazeSuite Software (www. mazesuite.com; Ayaz et al., 2008) on a Windows 7 computer, using procedures adapted from our previous study (Buckley and Bast, 2018). Given that we have described the environment used 
in this study in detail elsewhere (Buckley and Bast, 2018), we give only a brief overview of the materials to aid understanding of the task, and focus instead on the procedural details for the present EEG study.

The virtual environment was identical to that used in our previous study, and comprised of a circular grass lawn surrounded by a brown fence, beyond which eight cues (a tree, hot-air balloon, star, plane, tower, windmill, satellite and a planet), could be seen by participants and could be used for spatial orientation. These cues were varying distances from the fence, but distributed at regular $45^{\circ}$ intervals around the circular fence (Figure 1(a) and (b)).

The virtual arena was viewed from a first-person perspective, and participants were instructed to control movement through the environment using the four cursor keys on the keyboard with their right hand. Presses on the 'up', 'down', 'left' and 'right' keys permitted the participant to move forwards, backwards and to turn (rotate) counter-clockwise and clockwise, respectively. Trials started with the participant being placed in the environment facing the perimeter fence. As described elsewhere (Buckley and Bast, 2018), the environment was modelled on a rodent watermaze (Bast et al., 2009) and, thus, travelling from the start location to the opposite side of the fence (i.e. the diameter of the circle) took approximately $10 \mathrm{~s}$.

During an experimental session, participants were instructed to search for an invisible target, 'William the Worm', a cover story that has been used successfully with children and adults of varying ages (Buckley and Bast, 2018; Buckley et al., 2015), and to remember the location of the target using the cues arranged around the fence. Each participant completed one session of 32 trials, in which William the Worm would be located at eight different locations, for four trials at each location (Figure 1(c)). To prevent the use of egocentric strategies to navigate to each target location, participants began each of the four trials to the same target location at one of four different start positions spaced evenly along the fence perimeter (the notional N, E, S and W of the environment). The sequence of start positions and target locations was the same for all participants.

The overall 32 trials (to eight different locations) that each participant completed were split equally over four experimental blocks (Figure 1(d)). Each block started with a 120-s resting state period to serve as a baseline for the EEG analysis, in which a fixation cross was shown on a black screen throughout. Subsequently, within each block, participants would find William the Worm at two different locations. For each location within a session, the participant was given four consecutive trials to find the target (in the same location), before they were notified that the location of the target had changed.

For most trials of the experiment, participants were either given feedback once they found the target, or the target was highlighted to them after $120 \mathrm{~s}$ by a white flag appearing at the goal location. Having navigated to the target location, participants received a congratulatory message ('Congratulations!!! You found William! Congratulations!!!') and initiated the next trial using the Enter key. However, in experimental blocks 2-4, probe trials were administered on the second trial at the second location. Here, unlike every other trial in the task, participants were not informed (and the trial did not end) when they crossed the target location. Instead, participants were allowed to move freely for $60 \mathrm{~s}$ in order to allow the measurement of 'search preference' (see section 'Behavioural data analysis'). Each probe trial ended after $60 \mathrm{~s}$ had elapsed, after which a message ('Keep looking for William!') was displayed on the screen for $1 \mathrm{~s}$, and then the next normal trial (i.e. trial 3) began automatically.

\section{Behavioural data analysis}

All data analysis was carried out in MATLAB. The time-series of participants' positions were therefore imported and temporally aligned with the recorded EEG signals. For primary analysis, we calculated the following path-length measures: (1) 'total path length' of their translational (not rotational) movements in planar Euclidean space during the trial while searching for the target and (2) 'path efficiency' as the ratio between the 'total path length' and the distance of their starting position to the target position, which varies substantially from trial to trial (see Figure 2). Data from the first location (i.e. the first four trials) were excluded from analysis since participants were still adapting to the task.

In addition, we calculated a 'search preference' measure, which has been shown to be particularly dependent on hippocampal function in rat studies using the watermaze DMP test (Bast et al., 2009; McGarrity et al., 2017; Pezze and Bast, 2012). In this study, we measured the 'search preference' for the correct location during the probe trial as the average distance the participant kept from the target throughout the probe trial (with lower average distance reflecting higher search preference). A similar measure has been used in previous watermaze studies in rodents (Gallagher et al., 2015), although a more common measure of search preference in rodent watermaze and human virtual maze studies is the time the subjects spent within a pre-defined region around the target, often expressed as a proportion of time spent in other parts of the maze (Buckley and Bast, 2018). The reasons we used the distance-to-target measure (which was highly correlated with the proportion of time spent near the target) in this study were (1) that it only depends on the target location, without the requirement to define a zone around it or comparison areas and (2) ease of implementation.

\section{EEG recording and analysis}

EEG data were recorded using an EasyCap 120 channel system (with three peripheral electrodes - two located at the outer canthi of the eyes for horizontal electrooculography (EOG) and one below the left eye for vertical EOG recording, EasyCap GmbH, Germany). Data were sampled at $1000 \mathrm{~Hz}$ and amplified with a Sensorium amplifier (Sensorium Inc., Charlotte, VT, USA). Electrode impedances were kept below $20 \mathrm{kOhm}$. For each session (i.e. two maze runs with four trials each, plus a resting state period at the beginning), a new data file was started. Electrode locations of individual participants were digitised using a three-dimensional (3D) optical camera for neuronavigation for transcranial magnetic stimulation (TMS) (Brainsight ${ }^{\circledR}$, Rogue Research Inc., Quebec, Canada).

All analyses were conducted using FieldTrip (Oostenveld et al., 2011) and custom-written analysis software in MATLAB. Continuous data from both the rest and maze periods were notchfiltered for power line noise at $50 \mathrm{~Hz}$, temporally aligned to the maze-data and then split into 1-s epochs (pseudo-trials) to facilitate the analysis. All data from one block (of which there were four per participant) were concatenated and checked for excessive artefacts using a summary statistic (total power calculated 
(a)

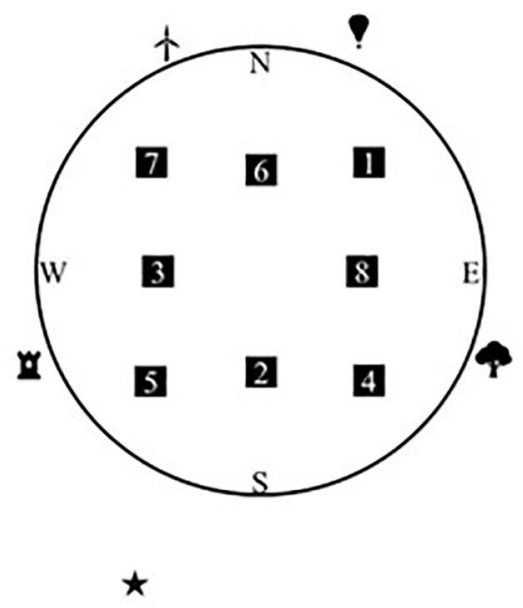

r (b)

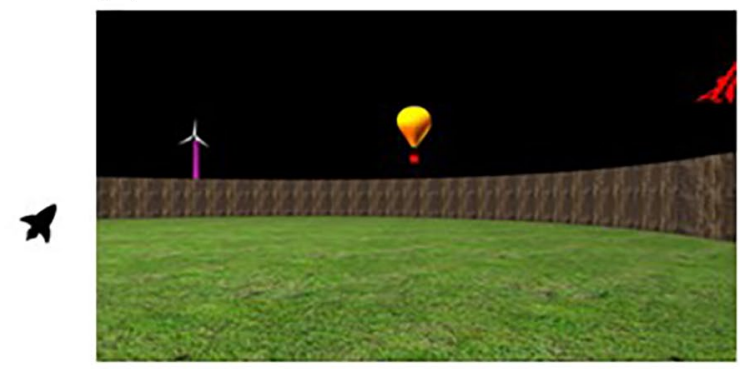

(c)

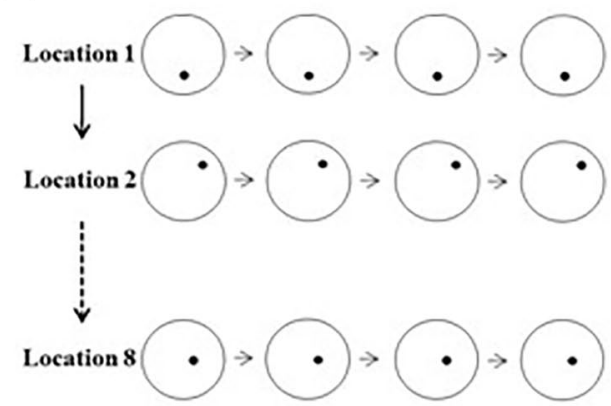

Self-paced trials, with trial $2 \mathrm{run}$ as a probe at location 4,6 , and 8

(d)

\begin{tabular}{cccccc}
$\begin{array}{c}\text { Experimental } \\
\text { phase }\end{array}$ & Target position & Trial 1 & Trial 2 & Trial 3 & Trial 4 \\
\hline \multirow{2}{*}{ Block 1 } & Location 1 & W & E & N & S \\
& Location 2 & S & N & E & W \\
\hline \multirow{2}{*}{ Block 2 } & Location 3 & E & W & S & N \\
& Location 4 & N & $\mathrm{S}^{*}$ & E & W \\
\hline \multirow{2}{*}{ Block 3 } & Location 5 & E & N & S & W \\
& Location 6 & N & $\mathrm{W}^{*}$ & S & E \\
\hline \multirow{2}{*}{ Block 4 } & Location 7 & S & E & N & W \\
& Location 8 & W & $\mathrm{N}^{*}$ & E & S \\
\hline
\end{tabular}

Figure 1. The virtual DMP task and the structure of the experimental sessions. (a) and (b) Participants were placed within a circular environment, with landmarks presented at varying distances from the circular wall, and were instructed to find a hidden target (see Figure 2 for an illustration of search paths). (c) In order to repeatedly test rapid place learning, the hidden goal (filled circles in panel (c)) moved after every four trials, and was placed at eight different locations during an experimental session (filled squares in panel (a)). (d) Each participant completed one experimental session split into four blocks. Each block started with a 2-min resting state period, followed by two sets of four navigation trials. During each set of four trials, the goal was placed in the same location, and participants began one trial from each of the notional cardinal points of the environment. Participants were then instructed that the goal had moved, and completed another set of four trials navigating to the new location. In trial 1 , participants could not know the location of the target and had to search for it. In trials 2-4, the participants could use the memory of the location acquired during trial 1 in order to navigate to the target efficiently. At locations 4, 6 and 8, the second of the four navigation trials was ran as a probe trial (marked by an *), during which no feedback was given when participants crossed the target location. Probe trials continued for $60 \mathrm{~s}$, during which participants' ‘search preference' for correct location could be measured. EEG was recorded continuously throughout the entire session. 


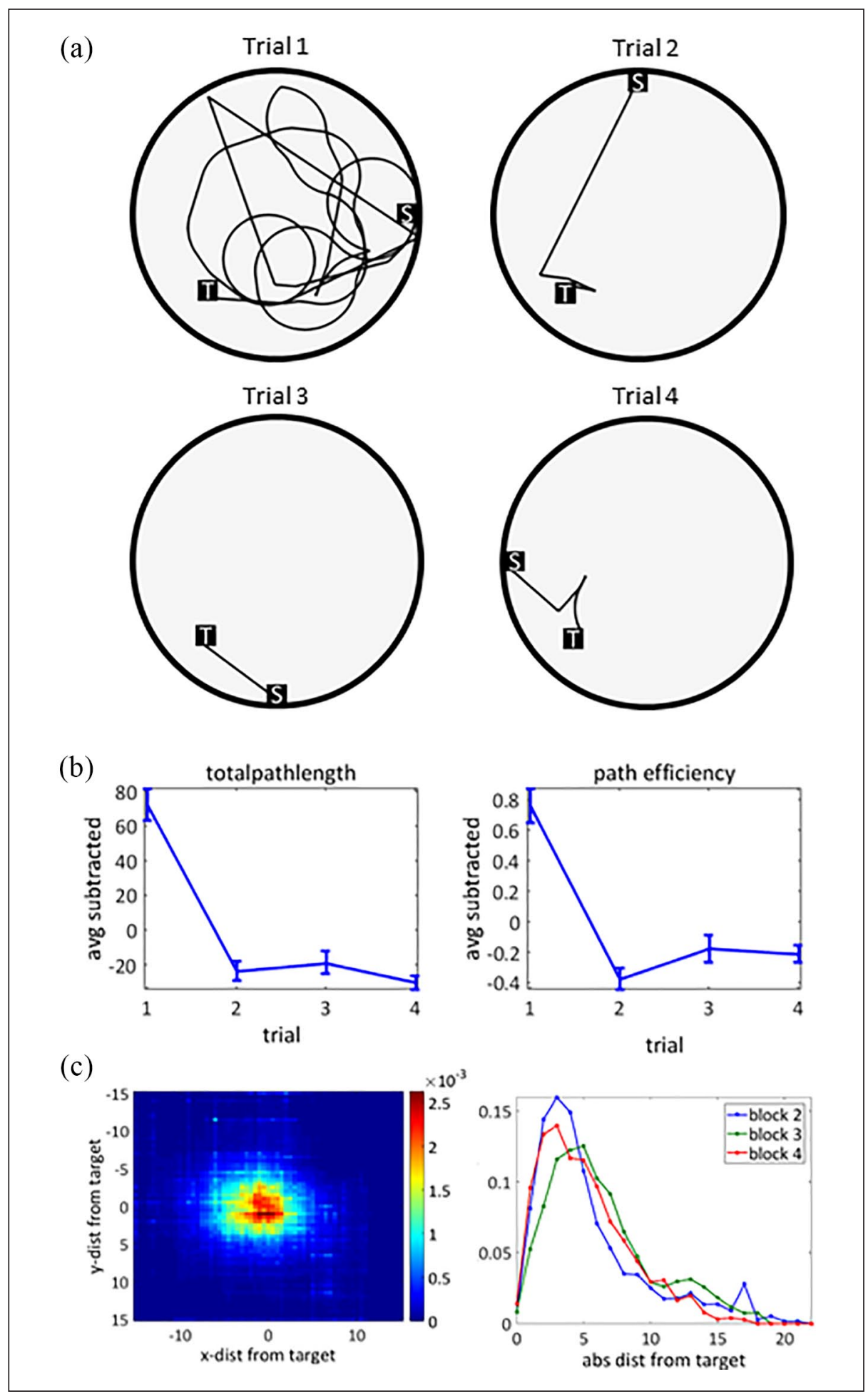

Figure 2. Behavioural performance on the virtual DMP test. (a) Illustration of one participant's search paths during the four trials to one location. (b) Path-length measures as a function of trial, averaged across all locations and participants. Left: total path length is the entire translational distance (without rotations) travelled by the participant; numbers represent (arbitrary) 'maze units' as distance measure. Right: path efficiency is path length normalised to the distance of starting position to target location. Averages of each participant across trials have been subtracted; error bars thus represent standard error of mean condition differences across participants, that is, confidence intervals. (c) Left: average heat map of participant locations during probe trials, with the target location at 0,0 . Colour code represents the average normalised frequency as a function of spatial location. Right: histogram of locations for the three different probe trials in sequence of occurrence. Numbers on $x$-axis represent the spatial distance from target in 'maze-units'; $y$-axis represents the normalised frequencies. 
Table 1. EasyCap electrode (Elec) and fiducial (nasion, NAS; left/right pre-auricular, LPA/RPA) positions in MNI coordinates (units: mm, rounded)..

\begin{tabular}{|c|c|c|c|c|c|c|c|c|c|c|c|}
\hline Elec & $x$ & $y$ & $z$ & Elec & $x$ & $y$ & $z$ & Elec & $x$ & $y$ & $z$ \\
\hline NAS & -7 & 82 & -44 & 38 & -82 & 6 & -12 & 78 & 56 & -51 & 76 \\
\hline LPA & -85 & -15 & -44 & 39 & -79 & 12 & 22 & 79 & 75 & -54 & 47 \\
\hline RPA & 84 & -17 & -42 & 40 & -65 & 22 & 50 & 80 & 76 & -65 & 2 \\
\hline 1 & -37 & 80 & -7 & 41 & -41 & 23 & 75 & 81 & -77 & -58 & 16 \\
\hline 2 & -8 & 88 & 0 & 42 & -9 & 30 & 86 & 82 & -71 & -57 & 45 \\
\hline 3 & 25 & 85 & -6 & 43 & 24 & 24 & 85 & 83 & -53 & -61 & 71 \\
\hline 4 & -40 & 76 & 5 & 44 & 55 & 23 & 63 & 84 & -31 & -60 & 89 \\
\hline 5 & -20 & 83 & 14 & 45 & 79 & 15 & 31 & 85 & 2 & -64 & 94 \\
\hline 6 & 10 & 86 & 13 & 46 & 82 & 6 & -7 & 86 & 36 & -67 & 84 \\
\hline 7 & 29 & 81 & 13 & 47 & -82 & -3 & 7 & 87 & 60 & -73 & 55 \\
\hline 8 & -60 & 60 & -17 & 48 & -76 & 2 & 42 & 88 & 70 & -72 & 24 \\
\hline 9 & -40 & 73 & 22 & 49 & -55 & 5 & 70 & 89 & -73 & -70 & 4 \\
\hline 10 & -25 & 79 & 29 & 50 & -25 & 8 & 91 & 90 & -68 & -73 & 32 \\
\hline 11 & -8 & 81 & 33 & 51 & 6 & 11 & 93 & 91 & -58 & -75 & 53 \\
\hline 12 & 11 & 82 & 32 & 52 & 40 & 6 & 82 & 92 & -42 & -73 & 74 \\
\hline 13 & 30 & 77 & 29 & 53 & 70 & 2 & 56 & 93 & -17 & -78 & 81 \\
\hline 14 & 58 & 68 & -8 & 54 & 84 & -7 & 14 & 94 & 14 & -81 & 82 \\
\hline 15 & -56 & 61 & 13 & 55 & -84 & -18 & -7 & 95 & 39 & -84 & 66 \\
\hline 16 & -50 & 60 & 33 & 56 & -81 & -15 & 24 & 96 & 55 & -89 & 37 \\
\hline 17 & -27 & 70 & 45 & 57 & -70 & -8 & 58 & 97 & 62 & -89 & 7 \\
\hline 18 & 8 & 72 & 49 & 58 & -42 & -5 & 85 & 98 & -58 & -85 & 35 \\
\hline 19 & 40 & 65 & 41 & 59 & -10 & -7 & 98 & 99 & -49 & -86 & 51 \\
\hline 20 & 52 & 64 & 23 & 60 & 25 & -7 & 92 & 100 & -29 & -89 & 64 \\
\hline 21 & -75 & 32 & -14 & 61 & 61 & -11 & 71 & 101 & 2 & -95 & 62 \\
\hline 22 & -68 & 44 & 9 & 62 & 82 & -17 & 35 & 102 & 29 & -96 & 55 \\
\hline 23 & -58 & 46 & 38 & 63 & 86 & -23 & 0 & 103 & 38 & -98 & 42 \\
\hline 24 & -39 & 52 & 56 & 64 & -84 & -32 & 15 & 104 & -58 & -93 & 13 \\
\hline 25 & -6 & 60 & 64 & 65 & -77 & -26 & 48 & 105 & -41 & -95 & 44 \\
\hline 26 & 15 & 60 & 64 & 66 & -57 & -23 & 75 & 106 & -31 & -98 & 46 \\
\hline 27 & 45 & 54 & 50 & 67 & -31 & -22 & 93 & 107 & -13 & -101 & 50 \\
\hline 28 & 64 & 49 & 25 & 68 & 8 & -28 & 101 & 108 & 6 & -100 & 51 \\
\hline 29 & 75 & 39 & -3 & 69 & 43 & -31 & 90 & 109 & 19 & -103 & 44 \\
\hline 30 & -77 & 26 & 1 & 70 & 72 & -37 & 60 & 110 & 38 & -109 & 9 \\
\hline 31 & -70 & 32 & 29 & 71 & 83 & -46 & 14 & 111 & -44 & -101 & 25 \\
\hline 32 & -55 & 31 & 57 & 72 & -82 & -50 & -3 & 112 & -30 & -105 & 31 \\
\hline 33 & -27 & 45 & 71 & 73 & -80 & -43 & 30 & 113 & 3 & -108 & 35 \\
\hline 34 & 3 & 45 & 78 & 74 & -67 & -40 & 63 & 114 & 18 & -108 & 30 \\
\hline 35 & 39 & 41 & 66 & 75 & -46 & -41 & 85 & 115 & -42 & -106 & 10 \\
\hline 36 & 61 & 38 & 45 & 76 & -14 & -44 & 98 & 116 & -14 & -113 & 18 \\
\hline 37 & 78 & 30 & 12 & 77 & 23 & -42 & 97 & 117 & 12 & -114 & 14 \\
\hline
\end{tabular}

for each trial and electrode) provided by $\mathrm{ft}$ rejectvisual. A principal component analysis (PCA) was then computed on these data and eye-blink components and obvious artefact components were removed from the data. A final inspection of any residual artefacts was carried out, using, again, the summary statistic provided by $\mathrm{ft}$ rejectvisual.

In order to have a reasonable separation of signals from different sources, for example, motor cortex, temporal lobe and occipital cortex, we conducted all data analyses in the source space using a beamformer transformation (Gross et al., 2001; Van Veen et al., 1997). To this end, we used the boundary element volume conductor model (BEM) of the segmented standard
Montreal Neurological Institute (MNI) brain as implemented in the FieldTrip toolbox (Oostenveld et al., 2011; Oostenveld et al., 2003). Average digitised electrode positions were aligned to the head surface (based on MNI brain, see Table 1) and the leadfields were calculated for a 3D grid covering the entire (MNI) brain with a spatial resolution of $1 \mathrm{~cm}$.

To calculate the source reconstructed power spectra with a good signal-to-noise ratio and in a computationally efficient way, we used the following approach: a time-domain linearly constrained maximum variance beamformer (Van Veen et al., 1997) was calculated on low-pass filtered (cut-off $30 \mathrm{~Hz}$ ) data from all epochs including the resting state period, using the covariance 
matrix from all these epochs concatenated across the four blocks and the leadfield matrix. This approach gives particularly robust filters (Bauer et al., 2012b; Bauer et al., 2014; Litvak et al., 2010). To obtain source estimates of power spectral density estimates, we then projected the real part (not the imaginary) of the ('sensor-level') cross-spectral density matrix (calculated in $1 \mathrm{~Hz}$ steps over $1 \mathrm{~s}$ epochs using a Hanning taper), averaged for all epochs of a particular trial type (resting state, trial 1, trial 2 exclusive probe trials, trial 3 , trial 4 and probe trial) through the beamformer.

This gave thus trial-specific estimates of power spectral density $(2-15 \mathrm{~Hz}$ in steps of $1 \mathrm{~Hz})$ for each of the 2015 grid points in the brain ( $1 \mathrm{~cm}$ regular grid $)$ - separately for resting state, trial 1 , trial 2, trial 3, trial 4 and probe trials, averaged across locations. Regarding the choice of spatial resolution and frequency range, see the next section.

\section{Statistical analyses of EEG correlates of behaviour}

The mere comparison of the navigation trials on the virtual DMP task versus rest is confounded by motor activations due to button presses, as well as dynamic visual input and enhanced attentional load during the navigation trials. Hence, this comparison is of limited suitability to reveal any memory-related activations.

In order to do so, we sought to correlate EEG power spectral density estimates with behavioural performance measures in the task. We chose the following approaches: (1) use the individual difference in path efficiency between trial 2 and trial 1 as a predictor of individual EEG activity during trial 1 to potentially reveal encoding processes; (2) use the same behavioural measure as a predictor of individual EEG activity during trial 2 to reveal retrieval/expression processes; (3) use the search preference for the target location, as reflected by average distance to target, during probe trials as predictor of EEG activity in probe trials to reveal retrieval/expression processes and (4) use the same behavioural variable (average distance to target during probe trial) to predict EEG activity during trial 1 to reveal encoding processes. Further control analyses following the same logic are described in section 'Results'.

We aimed to perform a data-driven analysis rather than aiming to confirm a priori hypotheses, and thus constraining our analysis on particular frequencies or regions of interest (also given the leakage of spatial filters, particularly with a canonical forward model). Given the large number of grid points (or 'voxels') covering the brain and the multiple frequencies involved, we corrected for the multiple comparison problem (for massunivariate testing), using a cluster-permutation approach (Maris and Oostenveld, 2007).

The frequency range and spatial resolution were chosen with the following rationale:

1. To provide a compromise between statistical sensitivity (power) when correcting for multiple comparisons with mass-univariate analyses and to cover a reasonable range with sufficient specificity.

2. Regarding the beamformer spatial resolution, although $1 \mathrm{~cm}$ is on the lower side, the use of a canonical volume conductor model (in contrast to individual ones based on segmented individual MRIs of participants' brains) and regularisation of the covariance matrix would likely prevent a higher effective spatial resolution anyway.

3. Regarding the frequency range, the lower bound was chosen to be just above the Rayleigh frequency, whereas the upper bound was chosen as to encompass a reasonably broad range of frequencies, including and extending beyond the low-frequency MTL oscillations that have been implicated in spatial navigation and memory (Ekstrom and Watrous, 2014) and that were the focus of this study, without making the statistical tests too insensitive.

Mass-univariate regression analyses were conducted using the behavioural metrics as a predictor variable and the EEG power of the particular trial type as the criterion with individual participants and blocks being units of observation; within- and between-subject variance was thus pooled in this approach to harvest as much of behavioural variation as possible. Since relatively large amplitude differences existed between participants, possibly related to impedances, the data were a priori normalised using the following approach: the individual (participant specific) source estimates of power spectral density were divided by the (individual) squared average of the square root of all power spectral density estimates across all frequencies and grid points during the 2-min resting state periods prior to each block

$$
S_{\text {task }}^{2}(i, f, v)=\frac{S_{\text {task }}^{2}(i, f, v)}{\left(\sum_{v}^{M} \sum_{f}^{K} S_{\text {rest }}(i, f, v)\right)^{2}}
$$

where $i$ is the index to the participant, $f$ is the index for $K$ frequencies, $v$ is the index for $M$ grid points (or voxels), and $S^{2}$ is the power spectral density estimate (i.e. the squared estimate of the complex Fourier coefficient). The cluster-permutation approach (Maris and Oostenveld, 2007), briefly summarised, was applied as follows: spatio-spectral clusters (in three spatial plus a spectral dimension) were formed of adjacent data points that survived the alpha-threshold of $p<0.01$ for two-sided testing on the regression coefficient. The cumulative $t$-value of the regression coefficients within each cluster was then calculated. This calculation for the real data was then repeated for 1000 randomised data sets, where, for each combination of frequency bin and spatial grid point (='voxel'), the associated behavioural parameter was shuffled randomly across observations (participants). A histogram of the maximum cluster metric for each randomisation was formed, serving as the reference, or null distribution. For those clusters of the real data for which the aggregated statistic was above the $5 \%$ percentile of this reference distribution, the omnibus null hypothesis can be rejected.

\section{Principal component control analysis}

As a control analysis, to stay closer to the recorded raw data and rely less on the algorithms that provide the inversion of the EEG data into brain space (beamformer source analysis), we additionally conducted an analysis where we projected the EEG raw data into the leading principal components for increased 
signal-to-noise ratio. PCA is a transformation of a data space into orthogonal new variables. It is, unlike a beamformer source analysis, free of assumptions about the biophysical origin of the electrical signals and independent of the validity of a volume conductor model.

To this end, the raw data of all participants (individual channels and every third trial to reduce memory) were $z$-transformed and then pooled together; a PCA was then computed on those trials to ensure all participants shared the same component structure. These data were then frequency analysed in the same way as for the beamformed data. Data (EEG and behavioural) of probe trials were then split into quintiles, sorted by the average distance to target, and the highest quintile was compared to the lowest quintile by means of a $t$-test for independent samples. In addition, the rest periods preceding the respective probe trials were compared in the same way.

\section{Results}

\section{Behavioural data: one-trial place learning on the virtual DMP test}

Figure 2(a) shows examples of the search paths taken by one participant during the four trials to one location. On trial 1, the participant searches for the new (unknown) target location, in this example in a mainly circular pattern (for other search strategies during trial 1, see Buckley and Bast, 2018, Figure 2). In subsequent trials, this search path is typically markedly more direct (i.e. shorter), reflecting one-trial place learning and leading to the behavioural summary statistics shown in Figure 2(b), left. What can also be appreciated from Figure 2(a) is that the distance of initial starting position in the maze to target varies markedly across trials, target locations and blocks (although this is kept constant across participants). To remove this variability, total path length was normalised to this initial distance and presented as 'path efficiency' (Figure 2(b), right). The latter metric effectively expresses the 'straightness' of the path taken to the target. Total path length was significantly shortened with higher trial numbers $\left(F(3,20)=47.85, p<10^{-8}\right)$, as was path efficiency $(F(3,20)=24.45$, $p<10^{-6}$ ), with a sharp reduction from trial 1 to 2 , reflecting onetrial place learning. For all measures, the average of each participant across conditions was first subtracted to make the standard errors of the mean (SEMs) interpretable regarding statistically significant differences (within-subject design).

When the second trial to a new location was run as a probe trial, where participants roamed around for $60 \mathrm{~s}$ without being given feedback about the target location, participants typically showed marked search preference for the target location by moving around in close proximity to the target (Figure 1(c)), reflecting one-trial place learning. Overall, both path-length measures and the search preference measure revealed marked one-trial place learning, similar to previous DMP studies in rodents and humans (Buckley and Bast, 2018).

\section{Correlations between behaviour and EEG activity}

Since the comparison between maze trials $1-4$, on one hand, and rest periods, on the other hand, confounds mnemonic processes with motor outputs, visual input and attentional demands, we focused on the analysis of inter-individual differences in behaviour and EEG parameters. All analyses were performed in 3D source space, using a regular $1 \mathrm{~cm}$ grid, based on a canonical forward model (MNI brain).

\section{Reductions in path-length measures from trial 1 to trial 2 show no significant correlations with EEG activity}

As a first step, we used the difference in path length and in path efficiency, respectively, between trial 1 and trial 2 as a predictor variable for EEG activity in either trials 1 (encoding) or in trials 2 (retrieval/expression, where these were not probe trials). The cluster analysis correcting for multiple comparison across space and frequency yielded no results that crossed the significance threshold (all $p>0.3$ ), both for trials 1 and trials 2 . Moreover, lowering the univariate clustering threshold to $p<0.05$ did not change this result. We interpret this to be due to the comparably small variability in the spatial distance measures (see Figure 2(b)).

\section{Negative correlations of theta oscillatory activity with distance to target - potential correlates of encoding and retrieval/ expression processes}

We next turned to the 'average distance to the target' during the 'probe trial', reflecting 'search preference' for the target location, which has been shown to be particularly dependent on hippocampal function in rat studies (Bast et al., 2009; McGarrity et al., 2017; Pezze and Bast, 2012). We used the average distance to the target during the three probe trials as a predictor variable for EEG data during both the same probe trial itself (i.e. reflecting retrieval/expression processes), as well as for the EEG data during the corresponding trial 1 (i.e. reflecting the encoding process). This analysis revealed negative correlations of oscillatory activity during probe trials (retrieval/expression) and during trial 1 (encoding) with average distance to target during probe trials (i.e. oscillatory activity predicted higher search preference), which were specific with respect to frequency and brain region (Figure 3(a)-(d)).

Figure 3(a) shows the spectrum of the thresholded statistics for the correlation between probe trial EEG activity and average distance to target during the probe trials, that is, during retrieval/ expression. Only data points that 'survived' the omnibus significance threshold of $p<0.05$ (two-sided) on the cluster level have values above zero, weighted by the number of data bins that reached significance - that is, the strength of the spectral statistics depends on the effect size in the respective frequency bin, as well as on the number of spatial grid points that are significant at that particular frequency, and thus reflects a weighted average. There was a negative theta peak $(6-8 \mathrm{~Hz})$, indicating that enhanced theta power correlates with a smaller average distance to the target, and thus more accurate one-trial place memory performance. Figure 3(b) shows the spatial signature of this cluster (significant at $p<0.05$, two-sided and corrected) of the correlation of theta-activity during the probe trial with memory performance (average distance to target during probe trial); brain areas contributing to the significant correlation encompassed 


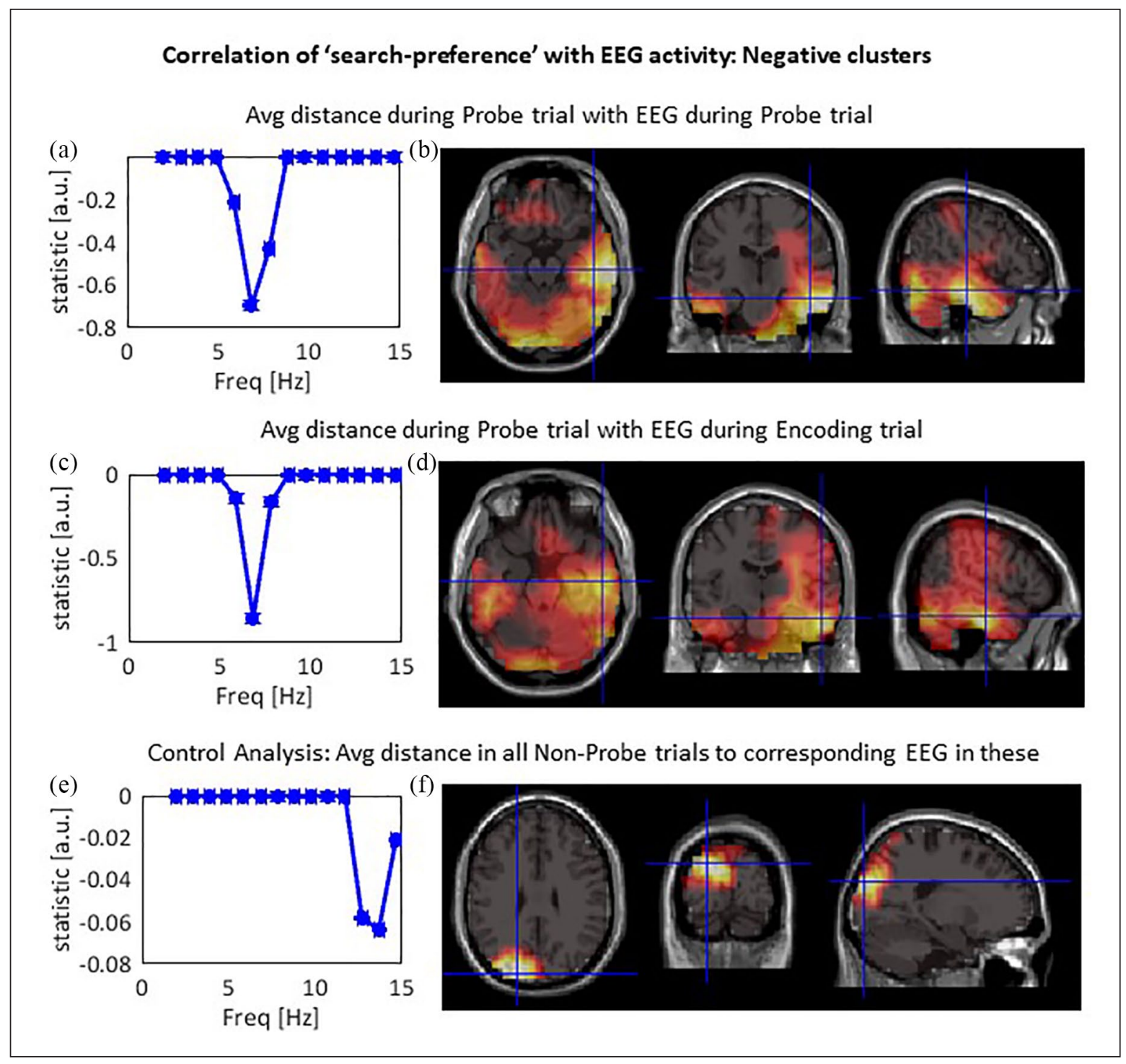

Figure 3. Negative correlations between oscillatory EEG activity and distance to target. (a)-(d) Negative correlations of EEG activity during probe trials (retrieval/expression) and trial 1 (encoding) with average distance to target during probe trial. Crosshairs in brain topographies show the location of cross-section planes (chosen to provide good overview). (a) Frequency spectrum of the omnibus significant cluster for the probe trial, correcting for multiple comparisons across frequencies and three spatial dimensions; higher theta-band activity during the probe trial is correlated with smaller distance to target. (b) Axial, coronal and sagittal views of the thresholded cluster (brain activations are sign-inverted to frequency spectra): activation is centred in (predominantly right) temporal cortex, inferior occipital cortex or cerebellum and striatum, forming one large cluster. (c) and (d) Same as (a) and (b), but now for the oscillatory activity during trial 1 (encoding); note the spectral and spatial similarity of the effects. (e) and (f) As a control analysis, the average distance during all non-probe trials was correlated to the EEG activity in the corresponding trials (to test for potential confounds, for example, related to sensorimotor factors). The largest cluster of this analysis was a peak in occipital alpha/beta activity (also sign-inverted) that did, however, not reach omnibus significance. All colour scales here show $t$-values averaged across the whole frequency domain (including frequencies where no significant correlation was found), with dark red corresponding to the lowest and white to the highest $t$-values, hence associated numeric values are arbitrary.

the temporal lobes, particularly in the right hemisphere and including the hippocampus and parahippocampal area, inferior occipital areas and/or cerebellum, as well as the right hemispheric striatum.

Figure 3(c) and (d) shows the same for the correlation of average distance to target during the probe trial and EEG activity in the immediately preceding trial 1 , that is, during encoding. Hence, here the EEG activity is not from the very same period during which the participant was moving across the maze, but it reflects neuronal activity of encoding, upon which the behavioural success of the probe trial depends. Note the remarkable similarity of the spectral and spatial pattern of the significant cluster $(p<0.05)$ to Figure 3(a) and (b), despite the EEG data being taken from a different period than the behavioural measure. 
To verify the specificity of these neuronal activation patterns to encoding and retrieval of one-trial place memory, we conducted the following three further control analyses:

1. We used the same average distance to target during the probe trial and correlated this to brain activity during corresponding trials 4 ; this failed to reveal any significant clusters (all $p>0.3$, positive or negative).

2. We then correlated the average distance to target from all trials (initially including probe trials) to their corresponding EEG activity. This revealed a significant cluster in occipital and temporal areas with a similar spectral profile $(p<0.05$, not shown) and spatially not too dissimilar to Figure 3(a)-(d). No positive clusters were found.

3. We repeated this analysis, but this time removing all probe trials, and thus correlating average distance from target to simultaneous EEG activity in trials 1, 3 and 4 of the second locations of each block (i.e. locations 4,6 and 8 ) and trials 1, 2, 3 and 4 from the first location of all blocks (3, 5 and 7) which never contained a probe trial, see Figure 1(b). This revealed a cluster that is, for comparison to Figure 3(a)-(d), depicted in Figure 3(e) and (f), showing a clear maximum in occipital cortex and a spectral peak in the alpha-band - but that did not reach omnibus significance $(p=0.14)$.

From this dissociation, we concluded that the highly similar outcomes shown in Figure 3(a)-(d) were specifically reflecting processes related to encoding and retrieving/expressing memory representations as behaviourally measured in terms of search preference during the probe trial.

\section{Positive correlations of alpha-oscillatory activity with distance to target - potential correlates of motor behaviour}

The cluster-permutation algorithm separates clusters showing positive and negative effects. In addition to the negative correlations of EEG activity with average distance to target, that is, patterns reflecting increased activity with better memory performance, our analysis also revealed several positive correlations of oscillatory activity during probe trials (retrieval/expression) and during trial 1 (encoding) with average distance to target during probe trials (i.e. more oscillatory activity predicted lower search preference), which were specific with respect to frequency and brain region (Figure 4). Figure 4(a) and (b) shows the positive correlation of alpha-activity $(12 \mathrm{~Hz})$ in motor cortex (left central sulcus over the hand area) during the probe trial to average distance from target during the same probe trial, although this did not reach omnibus significance $(p>0.2)$. Moreover, as shown in Figure 4(c) and (d), there was also a positive correlation for average distance to target with EEG activity during the (immediately preceding) trial 1 , that is, encoding. This positive correlation, with a similar alpha/beta $(12-14 \mathrm{~Hz})$ peak and a location covering large parts of parietal cortex contralateral to the navigation hand and, thus, sensorimotor areas related to visually guided hand movements (Buneo and Andersen, 2006; Pause et al., 1989), was of omnibus significance $(p<0.05)$. For an interpretation of these correlations, see further below.
The location of these positive clusters in motor cortex and parietal cortex, contralateral to the hand that participants were instructed to use for button presses to move through the maze, suggests that the correlations are movement-related since alphaactivity in the motor cortex (frequently also referred to as muactivity) is strongly associated with movement planning and execution (Pfurtscheller and Lopes da Silva, 1999). More specifically, they may reflect (1) direct movement-related activations of the motor cortex contralateral to the hand that participants were instructed to use for moving and (2) procedural or motor-learning that may facilitate retrieving the target location.

We tentatively (and speculatively) interpret these two results therefore in the following way:

1. For the non-significant (when considering multiple comparison correction) correlation during the probe trial itself (where EEG data reflect the same period as the behaviour, Figure 4(a) and (b)), this likely reflects a motor confound such that participants who remain closer to the target show less stationary movement patterns (more frequent turns and stop-and-go manoeuvres), requiring more frequent button presses and thus more alpha-suppression; thus, according to this explanation, enhanced distance to the target would coincide with fewer button presses and thereby less motor-related alpha-suppression, that is, higher alpha-activity.

2. For the omnibus significant positive correlation of alphaactivity during the encoding trial 1, (da Silva et al., 2014) with average distance from target during subsequent probe trials (Figure 4(c) and (d)), that is, impaired memory performance, we suspect that this may reflect that reduced movement (button presses), or exploration, during encoding results in weaker memory. This suggestion is consistent with the finding that the placement of rats onto the target location, instead of active swim trials, results in weaker memory in the watermaze DMP task (da Silva et al., 2014) and that volitional movements in human participants have been found to facilitate hippocampus-dependent memory in human participants (Voss et al., 2011).

In support of the implied relationships between movement during trial 1 or the probe, respectively, and average distance to target during probe trials, we found that the latter:

- Correlated negatively with the rate of button presses during probe trials at $r=-0.3(p<0.05)$; this means the closer participants remain to the target, the more frequently they press buttons on the same trial, probably because persistent searching close to the target location requires a high rate of button presses for frequent turns and stop and go (whereas continuous movement can be achieved by a low rate of button presses).

- Correlated negatively with the rate of button presses during the preceding trial 1 at $r=-0.2$, albeit non-significantly $(p>0.05)$.

- Correlated positively with the average speed of movement at $r=0.11$, although this correlation was far from significance $(p>0.35)$.

To summarise this: (1) parameters of motor behaviour during the probe trial correlate significantly with the memory-related 


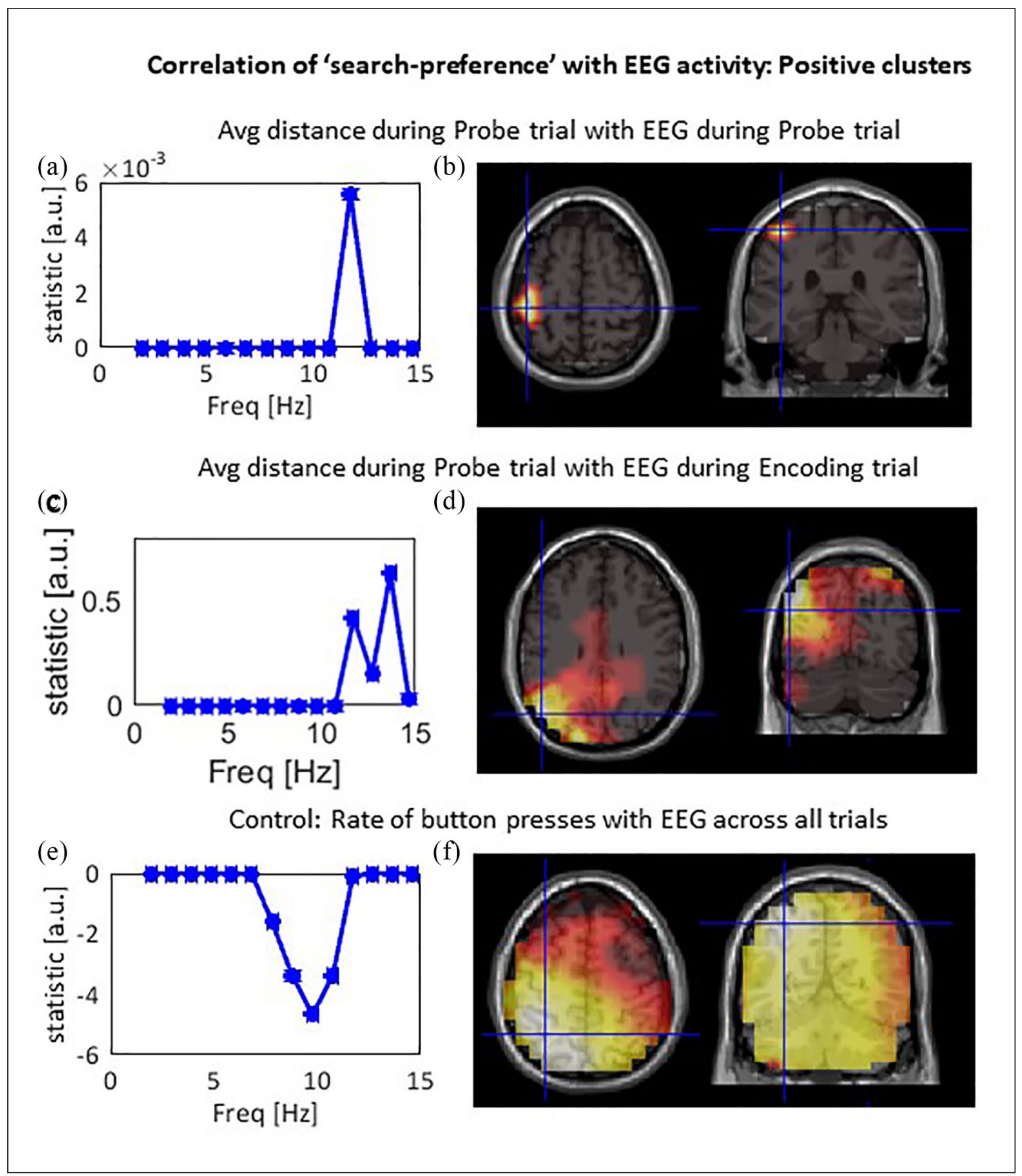

Figure 4. Positive correlations between oscillatory EEG activity and distance to target. (a)-(d) Positive correlations of EEG activity during probe trials (retrieval/expression) and trial 1 (encoding) with average distance to target during probe trials. Crosshairs in brain topographies show the location of cross-section planes (chosen to provide good overview). (a) The spectrum of this effect for the probe trial, while (b) its topography with a clear maximum over left primary sensorimotor cortex. This cluster, however, failed to reach omnibus significance. (c) The spectral distribution of the highly significant $(p<0.01)$ cluster for the encoding trial 1 , while $(d)$ its brain topography over left parietal areas. Since these effects, located over motor and sensorimotor regions, likely reflect motor-related patterns, a control analysis was conducted: the rate of button presses (see main text) was correlated with EEG activity across all trials. (e) The spectrum of this effect. Note that a higher rate of button presses (i.e. less stationary movement patterns) correlates with reduced alpha-activity (whereas in (a)-(d) the average distance from target, a value likely to be inversely related to button press rate was used). (f) The topography of this highly significant effect $(p<0.001)$, which is similar though more widespread than the effect in (b) and (d). All colour scales here show $t$-values averaged across the whole frequency domain (including frequencies where no significant correlation was found), with dark red corresponding to the lowest and white to the highest $t$-values, hence associated numeric values are arbitrary. 


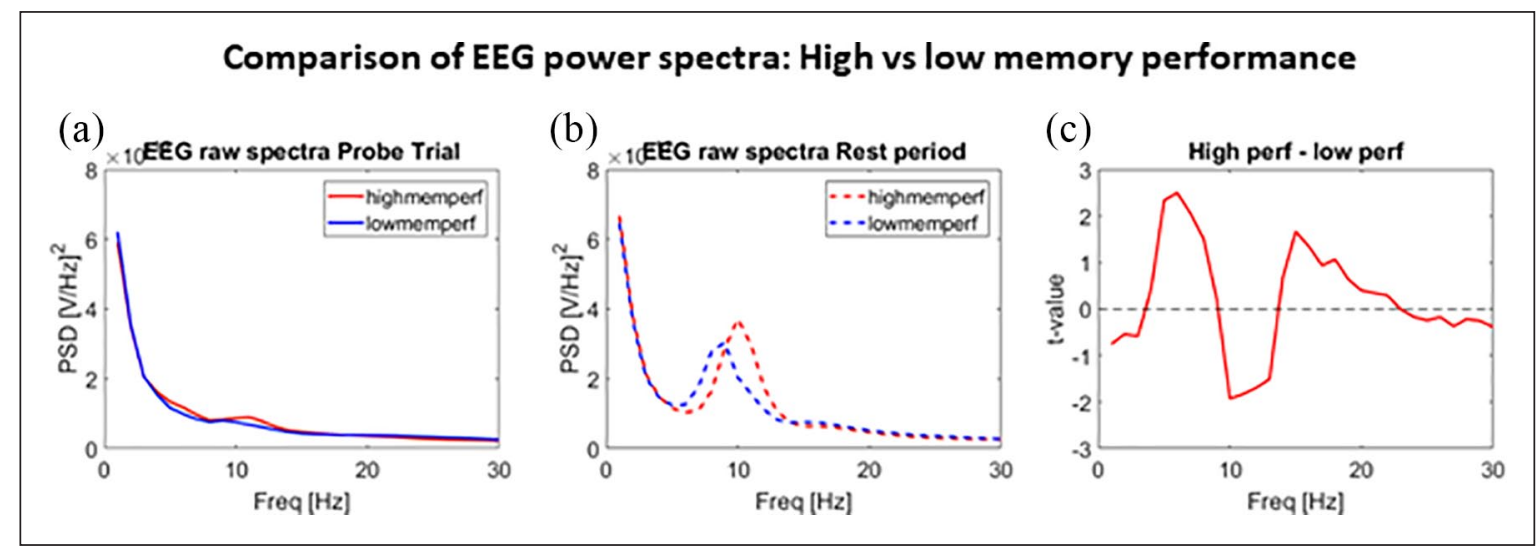

Figure 5. Principal component control analysis. (a) Averaged power spectra, not baseline corrected, in high-performing probe trials (red line) compared to low-performing probe trials (blue line). (b) The same for the resting state period immediately preceding the respective probe trials. (c) The results of the $t$-test for independent samples of baseline-corrected power during the probe trial.

average distance to target and are likely causing the effect in sensorimotor cortex in Figure 4(a) and (b) and (2) more speculatively at this point, procedural or motor-learning and possibly motor parameters of exploratory behaviour (da Silva et al., 2014) during encoding in trial 1 may represent the correlations shown in Figure 4(c) and (d).

This interpretation suggests that button presses across trials should negatively correlate with alpha-activity. Indeed, an analysis using the rate of button presses (and thus the variable that correlated to average distance to target during probe trial) in all trials as a predictor variable for EEG activity in all corresponding trials revealed a highly significant $(p<0.001)$ negative correlation with alpha-activity, involving large parts (probably due to the effect size) of left parietal and motor cortex and of occipital cortex (Figure 4(e) and (f)). The direction of this effect is consistent with the well-established finding that alpha-activity is associated with activation of neural tissue (Pfurtscheller and Lopes da Silva, 1999), that is, enhanced (sensori-) motor activity due to frequent button presses causing movement transients and therefore also non-stationary visual input. Importantly, no positive cluster was observed when button presses were used as predictor variable. Therefore, while movement-related factors are likely to account at least partially for the positive clusters in Figure 4(a)(d), there is no evidence that the memory network correlations presented in Figure 3 are a result of movement confounds.

\section{Principal component control analysis}

To further confirm the above findings concerning the association of low-frequency oscillations and one-trial place memory performance, we additionally show the raw power spectra for participants and trials with high versus low memory performance during the task and the rest period. We show this for principal components as these provide a data-transformation that boosts signal-to-noise ratio without making assumptions about the biophysical origin of the signals. Figure 5(a) shows the raw power spectra during the probe trial for high-achieving trials (with a small distance to target, that is, good memory performance) and for low-achieving trials (with a large distance to target, that is, poor memory performance), indicating higher theta- and alphapower in trials with good memory performance.
Figure 5(b) shows the same difference, now for the resting state period immediately preceding the respective probe trials, indicating that higher memory performance is associated with a higher alpha-peak frequency and amplitude during resting state period. Figure 5(c) then shows the comparison of the baselinecorrected power spectra for good versus bad probe trials (independent samples $t$-test). This comparison verifies that high memory performance is related to higher theta-, but lower alphapower changes (relative to baseline) during the probe trial.

\section{Discussion}

Combining the virtual DMP task with continuous EEG measures and using a correlational analysis unconstrained by a priori hypotheses on frequency bands or regions of interest, we have shown that better one-trial place memory performance (as reflected by average distance to target during probe trials) is predicted by theta oscillations within a largely right hemispheric network consisting of MTL (including parahippocampal area), striatum and inferior occipital cortex or cerebellum, both during encoding and during retrieval/expression. In addition, it emerged that enhanced memory performance was also correlated with reduced alpha-activity in sensorimotor cortical areas contralateral to the navigation hand during encoding (left parietal cortex) or probe trials (left motor cortex, although this correlation was not statistically significant), probably reflecting movementrelated factors that may at least partially be associated with memory performance.

\section{Comparison to previous MEG studies demonstrating hippocampal theta correlates of spatial memory and navigation}

Previous studies (Cornwell et al., 2008; Kaplan et al., 2012, 2014; Pu et al., 2017) have used MEG in combination with individual anatomical MRIs to demonstrate hippocampal theta contributions during encoding (Cornwell et al., 2008; Pu et al., 2017) and retrieval (Kaplan et al., 2012, 2014) of spatial memory and navigation in virtual mazes. In this study, the theta correlates of place memory performance were more pronounced in the right 
hemisphere (although some left temporal lobe activation was also evident), in line with hippocampal theta correlates reported by Kaplan et al. $(2012,2014)$ and Pu et al. (2017), and a wide range of evidence indicating that human spatial navigation memory is mainly associated with the right hippocampus (Burgess et al., 2002; Miller et al., 2018).

We, similar to Cornwell et al., (2008) and Pu et al., 2017) used an open-field navigation task, reverse-translated from a rodent watermaze paradigm, whereas Kaplan et al. (2014) and Kaplan et al. (2012) have relied on more structured tasks and used shorter epochs corresponding to specific encoding and retrieval periods to target temporal windows with an enhanced chance to detect hippocampal activation. Importantly, we used an analysis unconstrained by prior hypotheses on regions of interest or frequency bands to reveal theta originating from the MTL as a major neural mechanism underlying inter-individual differences in spatial learning. Interestingly, Cornwell et al. found a correlate of thetaactivity in hippocampus to the reduction in path length from trial 1 to trial 2. In this study, we did not find an omnibus significant oscillatory correlate of reductions in path-length measures from trial 1 to trial 2. However, note that: (1) in our data, there was relatively small inter-individual variation in the difference from trial 1 to trial 2, as can be appreciated by the small error bars in Figure 2 and (2) we may have used a more conservative statistical approach, also to deal with increased leakage of our spatial filters in EEG recordings based on a canonical forward model. Moreover, previous studies in humans and rats suggest that reductions in path-length measures from trial 1 and 2 and search preference on the DMP task may rely on partially dissociable neuropsychological mechanisms (Buckley and Bast, 2018). First, there is only a moderate correlation between these measures in both human participants and rats (Buckley and Bast, 2018). Second, in rats, only the search preference measure shows a significant decline with increasing retention delay between trial 1 and trial 2, reflecting forgetting (da Silva et al., 2014). Third, and most importantly, studies in rats show that the search preference measure is more strongly hippocampus-dependent, being more sensitive to disruption of hippocampal function by partial hippocampal lesions (Bast et al., 2009) and pharmacological manipulations (McGarrity et al., 2017; Pezze and Bast, 2012). Our finding that only the search preference measure showed theta correlates in the MTL is consistent with these previous findings.

\section{Reliability of our source reconstruction}

Analysing frequencies from 2 to $15 \mathrm{~Hz}$, as a trade-off between statistical power/sensitivity and ensuring a broad range, we were able to confirm - while correcting for multiple comparisons in 28,120 data points in frequency and spatial domain - that it was predominantly theta oscillations, in particular in the temporal lobe, that gave the best prediction of individual one-trial place memory performance on the virtual DMP task. Moreover, encoding and retrieval/expression correlates of memory performance were highly consistent, an issue we shall come back to further below. Although the anatomical locations of the theta correlates have to be interpreted with some caution on the basis of methodological limitations, this consistency between encoding and retrieval inspires a certain amount of confidence in their accuracy and spatial precision. Regarding the methodological constraints, two aspects should be mentioned as follows:
1. These results were obtained on the basis of a canonical (standard) forward model, based on the MNI brain and, therefore, based on averaged electrode positions for all participants (since individual positions on a standard brain and head surface were deemed meaningless); this imposes significant limitations on the spatial specificity, but perhaps also sensitivity of the beamformer solution (Troebinger et al., 2014). To address this issue, we applied a relatively high (noise-) regularisation of the covariance matrix. The comparably high spatial specificity of the functional images shown is also due to these showing a statistic that is thresholded for data points that survive a non-parametric significance threshold of $p<0.01$ and form part of a cluster that crosses the omnibus significance threshold (for details please refer to Maris and Oostenveld (2007)).

2. It would not be formally correct to ascertain on the basis of these results that a particular data point, which forms part of the omnibus significant cluster, drives this effect. For instance, the fact that the right striatum forms part of the cluster does not imply that this region is involved in spatial navigation memory at a confidence level given by the significance threshold corrected for multiple comparisons. This confidence level only applies to the entire cluster, not to each individual data point contributing to the shape of the cluster. However, the fact that (1) we chose a fairly conservative cluster significance criterion of $p<0.01$ and (2) we see strikingly similar activation patterns in two largely independent (for the part of the EEG data) comparisons, for encoding and retrieval, do instil confidence in these findings.

Finally, participants' movement and the dynamic visual input they received during task performance (as participants were moving relatively freely through the virtual environment) may have influenced our findings. However, it is highly unlikely that the theta correlates of one-trial place memory performance are explained by movement or dynamic visual input factors alone. None of the control analyses on movement parameters, nor a control analysis of our behavioural predictor variable of interest (average distance to target during probe trials) on unrelated trials yielded remotely similar theta correlates (see also next section on the alpha-correlates in sensorimotor regions of the brain). Hence, we conclude that movement or dynamic visual input effects are not the main factors to the effects we see in the putative network of temporal lobe, striatum and inferior occipital and/or cerebellar activation. Encoding of spatial memories in the real world also requires movements and dynamic visual input, such that avoiding this would be undesirable.

\section{The brain network supporting one-trial place memory performance}

Beyond the hypothesis-conform activation of the temporal lobe, including the hippocampal and parahippocampal region, we see further distinct activations in the right striatum and inferior occipital cortex and/or cerebellum - all at theta frequencies with a peak at $7 \mathrm{~Hz}$. Activity in these regions, at this frequency, correlated negatively with distance from the target - suggesting that higher theta-activity in these regions during both encoding and 
retrieval led to better one-trial place memory performance across individuals. Occipital involvement is consistent with the dependence of the task on visual input, and with previous studies implicating occipital theta-activity in performance of virtual spatial navigation tasks (Ekstrom, 2015) and in working memory tasks in both humans and animals (Fuentemilla et al., 2014; Lee et al., 2005).

With respect to cerebellum and striatum, it is evident that the quality of the anatomical forward model used in this study requires some caution and standard forward models developed for the cortical sheet are less suitable to detect such deep sources with cell geometries that may differ from the cortical sheet (Meyer et al., 2017). Nevertheless, our finding of a putative involvement of cerebellar theta-activity adds to an emerging literature that the cerebellum may provide input on self-motion and trajectory learning into the hippocampus, to facilitate the establishment of a cognitive map (Lefort et al., 2015), and is consistent with the suggestion that interactions between MTL and cerebellum may be coordinated by theta-activity (Watson et al., 2019). More directly, in one of the first, if not the first publication on electrophysiological hippocampal activation measured in humans in the context of a watermaze-like virtual maze, Cornwell et al. (2008) showed a highly similar cerebellar theta activation in their MEG recordings.

The striatal correlates observed in this study, in two separate conditions, are also consistent with some previous finding. Studies of human spatial navigation in a virtual maze have implicated the striatum and hippocampus (Doeller et al., 2008), and dopaminergic decline in the striatal-hippocampal circuit during ageing and Parkinson's disease has been associated with deficiencies in spatial navigation and memory (Thurm et al., 2016); these studies suggested that striatal mechanisms mainly serve cue-response strategies supporting spatial navigation, which are distinct from and complement hippocampal mechanisms of allocentric place representations. In addition, the human striatum has been implicated in performance on laboratory tests of hippocampus-dependent declarative memory (Scimeca and Badre, 2012). Moreover, rodent studies that combined behavioural testing with multi-site electrophysiological recordings (DeCoteau et al., 2007; Martin and Ono, 2000; van der Meer and Redish, 2011) or disconnection approaches (Devan and White, 1999; Floresco et al., 1997) support that hippocampal interactions with the striatum, especially mediodorsal and ventral striatum (the main recipients of hippocampal projections; (Humphries and Prescott, 2010; Voorn et al., 2004)), are important for rapid place-learning performance (reviewed in Bast (2011)). Studies in freely behaving rodents have also shown theta oscillations in the striatum and suggested that interactions between hippocampus and striatum, again mainly mediodorsal and ventral parts, are coordinated by theta oscillations in the context of spatial memory and navigation tasks (Berke et al., 2004; DeCoteau et al., 2007; Tort et al., 2008; van der Meer and Redish, 2011). Finally, in a recent study in rats, we found that functional inhibition targeting the ventral striatum disrupted one-trial place memory performance on the watermaze DMP task (Seaton et al., 2019).

The idea that theta oscillations support memory and particularly enhance the precision of spatial memory representations has a long history. Jensen and Lisman, (2000), for instance, found that when analysing the spike-time-series of place-field recordings, considering the position of the spikes in the phase of ongoing theta oscillations substantially boosted the decoding accuracy of the rat's position in the maze. Lee et al. (2005) found, in a visual working memory task, that during the encoding period, the delay activity was not characterised by an increase of spiking activity, but, instead, the spikes of those neurons carrying the stimulus representation strongly synchronise to the ongoing theta rhythm; this effect appears to be part of a network effect where phasesynchronisation of spikes in visual cortex to a prefrontal cortexdriven theta rhythm appears to be a major determinant on the success of working memory material retention (Liebe et al., 2012). Regarding the computational role of theta oscillations, one prominent idea is that theta oscillations facilitate and orchestrate spike-time-dependent plasticity (Lengyel et al., 2005; Sejnowski and Paulsen, 2006); theta oscillations in different brain regions may ensure that the inputs from different neuronal populations may be timed such that effective spike-time-dependent memory traces can be formed and retrieved. Neurophysiological studies and computational modelling suggest how theta oscillations in the hippocampus might play a mechanistic role during both encoding and retrieval (Hasselmo and Stern, 2014).

Overall, this study suggests that one-trial place memory performance may be supported by a brain network, coordinated by theta-activity and including the MTL, occipital cortex, cerebellum and striatum. The particular nature of the interactions between these regions in the theta range would perhaps be more suitably studied with further studies utilising individual and specialised forward models in the context of MEG recordings (due to enhanced MEG specificity to study neuronal interactions between different brain areas).

\section{Alpha-band correlates of DMP task performance}

Beyond the theta-band effects, we also observed that our measure of search preference during probes correlated with some activity in higher frequency bands, notably the sensorimotor alpha-band (Pfurtscheller et al., 1996), in sensorimotor areas contralateral to the hand that participants were instructed to use for navigation by button presses. This correlation was (omnibus) significant only in correspondence to the encoding trial and revealed a relatively broad cluster of parietal regions. For EEG activity during the probe trial (retrieval), this did not reach omnibus significance, but was localised very clearly to the hand area of the central sulcus (notably the critical $p$-value for individual data points was $p<0.01)$. Control analyses considered in section 'Results' suggested that these correlates reflect movement-related factors associated with task performance. The non-significant correlation of alpha-activity in the hand area during the probe trials with search preference during the probe trials likely reflected that participants with more precise spatial memory utilised more button presses to perform frequent turns and stop and go. The significant correlation of alpha-activity in parietal sensorimotor regions, which have been implicated in visually guided hand movements (Buneo and Andersen, 2006; Pause et al., 1989), during trial 1 with search preference during the probe trials may reflect that increased active exploration, or enhanced attention to one's movements (which would be expected to result in reduced alphapower (Bauer et al., 2012a; Jensen and Mazaheri, 2011)), during trial 1 boosts one-trial place memory. This suggestion is 
consistent with the finding in rats that placement of rats into the target location, instead of active swim trials to the target location, results in weaker memory in the watermaze DMP task (da Silva et al., 2014), and that in human participants, volitional movements have been found to facilitate hippocampus-dependent memory (Voss et al., 2011).

On top of this, in a control analysis comparing raw power spectra during probe trial and resting state period, it emerged that participants show larger alpha-amplitudes and higher alpha-peak frequency during the resting state period of trials with high onetrial place memory performance, compared to trials with low performance. Interestingly, previous studies have shown similar effects, relating higher alpha-peak frequency to better cognitive performance. Most notably, alpha-peak frequency has been shown to correlate with working memory performance (Posthuma et al., 2001), as well as with general intelligence (Grandy et al., 2013). However, alpha-peak frequency also serves as a predictor of low-level processing measures like pain sensitivity (Furman et al., 2018). While this may have, to some extent, contributed particularly to the negative correlation of alpha-power (in baseline-corrected data) with enhanced memory performance, the further investigation of the mechanisms behind this apparent alpha frequency effect in the present data set is beyond the scope of this article.

\section{Gamma oscillations and spatial navigation}

Previous studies - for example (Cornwell et al., 2014; Kaplan et al., 2014; Pu et al., 2018) - suggest that not only theta- but also gamma-oscillations play an important role for place memory performance, with distinct suggested roles of different hippocampal gamma frequencies for encoding and retrieval (Colgin et al., 2009) and a suggested mechanism for theta-gamma coupling (Lisman and Jensen, 2013). In this report, we focused on lowfrequency, including theta oscillations. These low-frequency oscillations have generally a much higher signal-to-noise ratio in human non-invasive recordings compared to gamma oscillations. In this study, where we correlate ongoing brain activity during a navigation tasks involving free viewing and (virtual) movement with a global behavioural measure (rather than an event-related design with repeated, and highly structured trials), the signal-tonoise ratio particularly of gamma oscillations, which overlap spectrally with muscular activity, can be expected to be particularly low. This is particularly true for EEG recordings that are known to suffer from a substantially lower signal-to-noise ratio compared to MEG recordings specifically in the higher frequency gamma-band (Muthukumaraswamy and Singh, 2013). In a preliminary analysis of the gamma-band, we were not able to identify a significant relationship between gamma oscillations and behavioural measures in our virtual DMP task. However, this does not preclude the possibility that such a relationship may be revealed, particularly if making use of sophisticated forward models that would enhance the accuracy of the source reconstruction and thereby the signal-to-noise ratio of (source) projected neural activity time courses, as well as generally more complicated analysis strategies. This, however, was not the purpose of this study; instead, the aim here was to show that it is possible to reveal inter-individual differences in oscillatory activity that predict differences in memory performance using (relatively) simple EEG recordings.

\section{Conclusion and future directions}

We have shown that, even when using a basic canonical forward model, surface EEG recordings in combination with the virtual DMP task can reveal the approximate neuroanatomy of a spatial memory system in the human brain on the basis of inter-individual variation in memory performance. This approach holds great potential for future studies on the potential oscillatory correlates of inter-individual differences, including in relation to agerelated changes in spatial memory (Coughlan et al., 2018; Driscoll et al., 2005; Hort et al., 2007; Lester et al., 2017) with a task that is suitable for different age groups (our own unpublished work with elderly participants and previous work using a related task in children (Buckley et al., 2015)). Some studies have already shown the great potential of the combination of surface EEG with similar tasks for clinical translation (Adams et al., 2020; Cornwell et al., 2010).

Of particular interest will also be the combination with neuropharmacological techniques to study the neurochemistry of such brain oscillations (Newman et al., 2012), as well as their importance for inter-individual differences in healthy (Richter et al., 2014) and clinical populations (Meng et al., 2018). The comparably low costs and the availability of EEG recordings may make this also suitable as a diagnostic tool for clinical practice. Regarding the question of the more mechanistic role of theta oscillations for interactions between different parts of the (putative) network that was revealed by our source analysis, these may be more suitably be addressed by MEG studies with sophisticated forward models (Meyer et al., 2017).

\section{Acknowledgements}

The authors thank Maazah Muhammad Ali, Zaariyah Hasan Bashir, Jessica Burrington, Irina Surducan, Abigail Webb and Desislava Abu Rashed for assistance with data collection.

\section{Declaration of conflicting interests}

The author(s) declared no potential conflicts of interest with respect to the research, authorship and/or publication of this article.

\section{Funding}

The author(s) disclosed receipt of the following financial support for the research, authorship and/or publication of this article: M.B. was supported by a Nottingham Research Fellowship.

\section{ORCID iDs}

Markus Bauer (iD https://orcid.org/0000-0001-6906-1678 Matthew Buckley (iD https://orcid.org/0000-0002-6879-9408

\section{References}

Adams RA, Bush D, Zheng F, et al. (2020) Impaired theta phase coupling underlies frontotemporal dysconnectivity in schizophrenia. Brain 143(4): 1261-1277.

Ayaz H, Allen SL, Platek SM, et al. (2008) Maze Suite 1.0: A complete set of tools to prepare, present, and analyze navigational and spatial cognitive neuroscience experiments. Behav Res Methods 40: 353-359.

Bast T (2007) Toward an integrative perspective on hippocampal function: From the rapid encoding of experience to adaptive behavior. Rev Neurosci 18(3-4): 253-281. 
Bast T (2011) The hippocampal learning-behavior translation and the functional significance of hippocampal dysfunction in schizophrenia. Curr Opin Neurobiol 21(3): 492-501.

Bast T, Wilson IA, Witter MP, et al. (2009) From rapid place learning to behavioral performance: A key role for the intermediate hippocampus. PLoS Biol 7(4): e1000089.

Bauer M, Kennett S and Driver J (2012a) Attentional selection of location and modality in vision and touch modulates low-frequency activity in associated sensory cortices. J Neurophysiol 107(9): 2342-2351.

Bauer M, Kluge C, Bach D, et al. (2012b) Cholinergic enhancement of visual attention and neural oscillations in the human brain. Curr Biol 22(5): 397-402.

Bauer M, Stenner MP, Friston KJ, et al. (2014) Attentional modulation of alpha/beta and gamma oscillations reflect functionally distinct processes. J Neurosci 34(48): 16117-16125.

Berke JD, Okatan M, Skurski J, et al. (2004) Oscillatory entrainment of striatal neurons in freely moving rats. Neuron 43(6): 883-896.

Braak H and Braak E (1995) Staging of Alzheimer's disease-related neurofibrillary changes. Neurobiol Aging 16(3): 271-278; discussion 284.

Buckley MG and Bast T (2018) A new human delayed-matching-toplace test in a virtual environment reverse-translated from the rodent watermaze paradigm: Characterization of performance measures and sex differences. Hippocampus 28(11): 796-812.

Buckley MG, Haselgrove M and Smith AD (2015) The developmental trajectory of intramaze and extramaze landmark biases in spatial navigation: An unexpected journey. Dev Psychol 51(6): 771-791.

Buneo CA and Andersen RA (2006) The posterior parietal cortex: Sensorimotor interface for the planning and online control of visually guided movements. Neuropsychologia 44(13): 2594-2606.

Burgess N, Maguire EA and O'Keefe J (2002) The human hippocampus and spatial and episodic memory. Neuron 35(4): 625-641.

Bush D, Bisby JA, Bird CM, et al. (2017) Human hippocampal theta power indicates movement onset and distance travelled. Proc Natl Acad Sci U S A 114(46): 12297-12302.

Colgin LL (2013) Mechanisms and functions of theta rhythms. Аnпи Rev Neurosci 36(8): 295-312.

Colgin LL, Denninger T, Fyhn M, et al. (2009) Frequency of gamma oscillations routes flow of information in the hippocampus. Nature 462(7271): 353-357.

Cornwell BR, Johnson LL, Holroyd T, et al. (2008) Human hippocampal and parahippocampal theta during goal-directed spatial navigation predicts performance on a virtual Morris water maze. $J$ Neurosci 28(23): 5983-5990.

Cornwell BR, Overstreet C and Grillon C (2014) Spontaneous fast gamma activity in the septal hippocampal region correlates with spatial learning in humans. Behav Brain Res 261(1): 258-264.

Cornwell BR, Salvadore G, Colon-Rosario V, et al. (2010) Abnormal hippocampal functioning and impaired spatial navigation in depressed individuals: Evidence from whole-head magnetoencephalography. Am J Psychiatry 167(7): 836-844.

Coughlan G, Laczo J, Hort J, et al. (2018) Spatial navigation deficits overlooked cognitive marker for preclinical Alzheimer disease? Nat Rev Neurol 14(8): 496-506.

da Silva BM, Bast T and Morris RG (2014) Spatial memory: Behavioral determinants of persistence in the watermaze delayed matching-toplace task. Learn Mem 21(1): 28-36.

DeCoteau WE, Thorn C, Gibson DJ, et al. (2007) Learning-related coordination of striatal and hippocampal theta rhythms during acquisition of a procedural maze task. Proc Natl Acad Sci U S A 104(13): 5644-5649.

Devan BD and White NM (1999) Parallel information processing in the dorsal striatum: Relation to hippocampal function. J Neurosci 19(7): 2789-2798

Doeller CF, King JA and Burgess N (2008) Parallel striatal and hippocampal systems for landmarks and boundaries in spatial memory. Proc Natl Acad Sci U S A 105(15): 5915-5920.
Driscoll I, Hamilton DA, Yeo RA, et al. (2005) Virtual navigation in humans: The impact of age, sex, and hormones on place learning. Horm Behav 47(3): 326-335.

Ekstrom AD (2015) Why vision is important to how we navigate. Hippocampus 25(6): 731-735.

Ekstrom AD and Watrous AJ (2014) Multifaceted roles for low-frequency oscillations in bottom-up and top-down processing during navigation and memory. Neuroimage 85(Pt2): 667-677.

Floresco SB, Seamans JK and Phillips AG (1997) Selective roles for hippocampal, prefrontal cortical, and ventral striatal circuits in radial-arm maze tasks with or without a delay. J Neurosci 17(5): $1880-1890$.

Fuentemilla L, Barnes GR, Duzel E, et al. (2014) Theta oscillations orchestrate medial temporal lobe and neocortex in remembering autobiographical memories. Neuroimage 85(Pt2): 2730-737.

Furman AJ, Meeker TJ, Rietschel JC, et al. (2018) Cerebral peak alpha frequency predicts individual differences in pain sensitivity. Neuroimage 167(15): 203-210.

Gallagher M, Burwell R and Burchinal M (2015) Severity of spatial learning impairment in aging: Development of a learning index for performance in the Morris water maze. Behav Neurosci 129(4): 540-548.

Grandy TH, Werkle-Bergner M, Chicherio C, et al. (2013) Individual alpha peak frequency is related to latent factors of general cognitive abilities. Neuroimage 79(1): 10-18.

Gross J, Kujala J, Hamalainen M, et al. (2001) Dynamic imaging of coherent sources: Studying neural interactions in the human brain. Proc Natl Acad Sci U S A 98(2): 694-699.

Hasselmo ME and Stern CE (2014) Theta rhythm and the encoding and retrieval of space and time. Neuroimage 85(Pt2): 656-666.

Herweg NA, Solomon EA and Kahana MJ (2020) Theta Oscillations in Human Memory. Trends Cogn Sci 24(6524): 208-227.

Hillebrand A and Barnes GR (2002) A quantitative assessment of the sensitivity of whole-head MEG to activity in the adult human cortex. Neuroimage 16(3Pt1): 638-650.

Hort J, Laczo J, Vyhnalek M, et al. (2007) Spatial navigation deficit in amnestic mild cognitive impairment. Proc Natl Acad Sci U S A 104(10): 4042-4047.

Humphries MD and Prescott TJ (2010) The ventral basal ganglia, a selection mechanism at the crossroads of space, strategy, and reward. Prog Neurobiol 90(4): 385-417.

Jensen O and Lisman JE (2000) Position reconstruction from an ensemble of hippocampal place cells: Contribution of theta phase coding. Journal of Neurophysiology 83(5): 2602-2609.

Jensen O and Mazaheri A (2011) Shaping functional architecture by oscillatory alpha activity: Gating by inhibition. Front Hum Neurosci 4(1): 186.

Kahana MJ, Sekuler R, Caplan JB, et al. (1999) Human theta oscillations exhibit task dependence during virtual maze navigation. Nature 399(2): 781-784.

Kaplan R, Bush D, Bonnefond M, et al. (2014) Medial prefrontal theta phase coupling during spatial memory retrieval. Hippocampus 24(6): 656-665.

Kaplan R, Doeller CF, Barnes GR, et al. (2012) Movement-related theta rhythm in humans: Coordinating self-directed hippocampal learning. PLoS Biol 10(2): e1001267.

Kramer JH, Schuff N, Reed BR, et al. (2004) Hippocampal volume and retention in Alzheimer's disease. J Int Neuropsychol Soc 10(4): 639-643.

Lee H, Simpson GV, Logothetis NK, et al. (2005) Phase locking of single neuron activity to theta oscillations during working memory in monkey extrastriate visual cortex. Neuron 45(1): 147-156.

Lefort JM, Rochefort C, Rondi-Reig L, et al. (2015) Cerebellar contribution to spatial navigation: New insights into potential mechanisms. Cerebellum 14(1): 59-62.

Lengyel M, Kwag J, Paulsen O, et al. (2005) Matching storage and recall: Hippocampal spike timing-dependent plasticity and phase response curves. Nat Neurosci 8(12): 1677-1683. 
Lester AW, Moffat SD, Wiener JM, et al. (2017) The aging navigational system. Neuron 95(5): 1019-1035.

Liebe S, Hoerzer GM, Logothetis NK, et al. (2012) Theta coupling between V4 and prefrontal cortex predicts visual short-term memory performance. Nat Neurosci 15: 456-462.

Lisman JE and Jensen O (2013) The theta-gamma neural code. Neuron 77(6): 1002-1016.

Litvak V, Eusebio A, Jha A, et al. (2010) Optimized beamforming for simultaneous MEG and intracranial local field potential recordings in deep brain stimulation patients. Neuroimage 50(4): 1578-1588.

McGarrity S, Mason R, Fone KC, et al. (2017) Hippocampal neural disinhibition causes attentional and memory deficits. Cereb Cortex 27(9): $4447-4462$

Maris E and Oostenveld R (2007) Nonparametric statistical testing of EEG- and MEG-data. J Neurosci Methods 164(1): 177-190.

Martin PD and Ono T (2000) Effects of reward anticipation, reward presentation, and spatial parameters on the firing of single neurons recorded in the subiculum and nucleus accumbens of freely moving rats. Behav Brain Res 116(1): 23-38.

Meng D, Li X, Bauer M, et al. (2018) Altered nucleus Basalis connectivity predicts treatment response in mild cognitive impairment. Radiology 289(3): 775-785.

Meyer SS, Rossiter H, Brookes MJ, et al. (2017) Using generative models to make probabilistic statements about hippocampal engagement in MEG. Neuroimage 149(1): 468-482.

Miller E (1973) Short- and long-term memory in patients with presenile dementia (Alzheimer's disease). Psychol Med 3(2): 221-224.

Miller J, Watrous AJ, Tsitsiklis M, et al. (2018) Lateralized hippocampal oscillations underlie distinct aspects of human spatial memory and navigation. Nat Commun 9(1): 2423.

Morris R (2007) Theories of Hippocampal Function. the Hippocampus Book. New York: Oxford University Press, pp. 581-714.

Morris RG, Garrud P, Rawlins JN, et al. (1982) Place navigation impaired in rats with hippocampal lesions. Nature 297: 681-683.

Muthukumaraswamy SD and Singh KD (2013) Visual gamma oscillations: The effects of stimulus type, visual field coverage and stimulus motion on MEG and EEG recordings. Neuroimage 69(1): 223-230.

Newman EL, Gupta K, Climer JR, et al. (2012) Cholinergic modulation of cognitive processing: Insights drawn from computational models. Front Behav Neurosci 6(13): 24.

O'Keefe J and Burgess N (1999) Theta activity, virtual navigation and the human hippocampus. Trends Cogn Sci 3(11): 403-406.

Oostenveld R, Fries P, Maris E, et al. (2011) FieldTrip: Open source software for advanced analysis of MEG, EEG, and invasive electrophysiological data. Comput Intell Neurosci 2011: 156869.

Oostenveld R, Stegeman DF, Praamstra P, et al. (2003) Brain symmetry and topographic analysis of lateralized event-related potentials. Clin Neurophysiol 114(7): 1194-1202.

Pause M, Kunesch E, Binkofski F, et al. (1989) Sensorimotor disturbances in patients with lesions of the parietal cortex. Brain 112(Pt6): $1599-1625$.

Pezze M and Bast T (2012) Dopaminergic modulation of hippocampusdependent learning: Blockade of hippocampal D1-class receptors during learning impairs 1 -trial place memory at a 30 -min retention delay. Neuropharmacology 63(4): 710-718.
Pfurtscheller G and Lopes da Silva FH (1999) Event-related EEG/MEG synchronization and desynchronization: Basic principles. Clin Neurophysiol 110(11): 1842-1857.

Pfurtscheller G, Stancak A and Jr Neuper C (1996) Event-related synchronization (ERS) in the alpha band - An electrophysiological correlate of cortical idling: A review. Int J Psychophysiol 24(1-2): 39-46.

Posthuma D, Neale MC, Boomsma DI, et al. (2001) Are smarter brains running faster? Heritability of alpha peak frequency, IQ, and their interrelation. Behav Genet 31(2): 567-579.

$\mathrm{Pu}$ Y, Cornwell BR, Cheyne D, et al. (2017) The functional role of human right hippocampal/parahippocampal theta rhythm in environmental encoding during virtual spatial navigation. Hum Brain Mapp 38(3): $1347-1361$.

$\mathrm{Pu}$ Y, Cornwell BR, Cheyne D, et al. (2018) High-gamma activity in the human hippocampus and parahippocampus during inter-trial rest periods of a virtual navigation task. Neuroimage 178(5): 92-103.

Richter N, Allendorf I, Onur OA, et al. (2014) The integrity of the cholinergic system determines memory performance in healthy elderly. Neuroimage 100(15): 481-488.

Scimeca JM and Badre D (2012) Striatal contributions to declarative memory retrieval. Neuron 75(3): 380-392.

Seaton A, Gwilt M, Hock R, et al. (2019) A role for the nucleus accumbens in the hippocampal learning-behaviour translation. British Neuroscience Association Abstract 25(PS045): 25045.

Sejnowski TJ and Paulsen O (2006) Network oscillations: Emerging computational principles. J Neurosci 26(2): 1673-1676.

Steele RJ and Morris RG (1999) Delay-dependent impairment of a matching-to-place task with chronic and intrahippocampal infusion of the NMDA-antagonist D-AP5. Hippocampus 9(2): 118-136.

Thurm F, Schuck NW, Fauser M, et al. (2016) Dopamine modulation of spatial navigation memory in Parkinson's disease. Neurobiol Aging 38(8): 93-103.

Tiesinga PH, Fellous JM, Jose JV, et al. (2001) Computational model of carbachol-induced delta, theta, and gamma oscillations in the hippocampus. Hippocampus 11(3): 251-274.

Tort AB, Kramer MA, Thorn C, et al. (2008) Dynamic cross-frequency couplings of local field potential oscillations in rat striatum and hippocampus during performance of a T-maze task. Proc Natl Acad Sci US A 105(51): 20517-20522.

Troebinger L, Lopez JD, Lutti A, et al. (2014) High precision anatomy for MEG. Neuroimage 86(100): 583-591.

van der Meer MA and Redish AD (2011) Theta phase precession in rat ventral striatum links place and reward information. $J$ Neurosci 31(8): 2843-2854

Van Veen BD, van Drongelen W, Yuchtman M, et al. (1997) Localization of brain electrical activity via linearly constrained minimum variance spatial filtering. IEEE Trans Biomed Eng 44(9): 867-880.

Voorn P, Vanderschuren LJ, Groenewegen HJ, et al. (2004) Putting a spin on the dorsal-ventral divide of the striatum. Trends Neurosci 27(8): 468-474.

Voss JL, Gonsalves BD, Federmeier KD, et al. (2011) Hippocampal brain-network coordination during volitional exploratory behavior enhances learning. Nat Neurosci 14(1): 115-120.

Watson TC, Obiang P, Torres-Herraez A, et al. (2019) Anatomical and physiological foundations of cerebello-hippocampal interaction. Elife 8: e41896. 